\title{
Beneficiation of Heat-Treated Crushed Brown Coal for Energy Production and Utilities
}

\author{
V.A. Moiseev, V.G. Andrienko, V.G. Piletskii, V.A. Donchenko \\ and A.I. Urvantsev
}

\begin{abstract}
The article deals with the problems of increasing the efficiency of electric separators through developing new approached to their design for upgrading of heat-treated crushed brown coal. The rational use of natural resources and efficient energy consumption are two basic requirements of EU Directive 2008/1/EC. It is expected that the techniques for brown coal pre-drying will result in increased energy efficiency of enterprises of up to 5\% and upgrading of heat-treated brown coal will increase energy efficiency by the same value. To upgrade heat-treated brown coal it is necessary to design new equipment including high-efficiency electric separators. It was earlier found that the maximum value of coal particle charge was provided in a corona-electrostatic separator, a lower level of coal particle charge was observed in a triboelectrostatic separator and the lowest level of coal particle charge was established in a plate-type electrostatic separator. The basic efficiency constraint for drum-type corona-electrostatic separators is the diameter of a collecting electrode and one operating area. To select the directions for increasing the productivity and efficiency in separating heat-treated crushed brown coal in drum-type corona-electrostatic separators the results of studies of changes in force vectors affecting its charged particles, nature of their motion in the electric field resulting in separated productsorganic and mineral components of brown coal, taking this into consideration the factors of the heating temperature, voltage across a corona-producing electrode (drum), diameter of a corona-producing electrode and its rotation speed, have been analyzed. The increased efficiency of a drum-type CES with a slight increase in its weight is associated with removing a constraint from design parameters - the diameter of a collecting electrode (drum). It is realized through the change of orientation of a collecting electrode from horizontal to vertical.
\end{abstract}

Keywords Heat-treated brown coal - Upgrading • Particle charge A corona-electrostatic separator - Efficiency • Vertical corona-producing electrode (drum)

\footnotetext{
V.A. Moiseev · V.G. Andrienko · V.G. Piletskii $(\bowtie)$ · V.A. Donchenko · A.I. Urvantsev "COMPOMASH-TEK" Company, Moscow, Russia

e-mail: pvg2000@yandex.ru

(C) The Author(s) 2018

K.V. Anisimov et al. (eds.), Proceedings of the Scientific-Practical Conference

"Research and Development - 2016", https://doi.org/10.1007/978-3-319-62870-7_34
} 


\section{Introduction}

The rational use of natural resources and efficient energy consumption are two basic requirements of EU Directive 2008/1/EC. It is expected that the techniques for brown coal pre-drying will result in increased energy efficiency of enterprises of up to $5 \%$ and upgrading of heat-treated brown coal will increase energy efficiency by the same value. To upgrade heat-treated brown coal it is necessary to design new equipment including high-efficiency electric separators. After performing theoretical and laboratory researches of processes of effecting upgrading of heat-treated crushed brown coal HTCBC it has been proven that the basis for changing electrical conductivity in particles of crushed brown coal, which improves the efficiency of upgrading, is its heat treatment. Thus, electrical conductivity for semiconductors and dielectrics increases when the temperature rises, separation of charged particles of HTCBC is improved due to their drying, classification and dedusting [1-3]. Figure 1 shows the dependence of the charge of coal particles of different sizes on the heating temperature. The researchers have identified the reasonability of heating up to $120-160{ }^{\circ} \mathrm{C}$ with electric separation of coal particles for effective separation of a mineral component, for example, fractions " $-0.5+0.0 ", 14 \%$ yield, $9.6 \%$ ash content.

Dependence of HTCBC charges and particles of non-heat-treated brown coal on the temperature is shown in Fig. 2. When particle sizes decrease the charge increases. Heating of particles up to $105^{\circ} \mathrm{C}$ is accompanied by condensation removal and reduced resistivity of a substance (from $10^{6}$ to $10^{3} \mathrm{M} \mathrm{Ohm}$ ) [4-6]. This results in increase of contact potential difference.

Before separating on drum-type separators sizing is recommended otherwise centrifugal forces, proportional to the cube of diameter of particles, can neutralize the effect of electric forces proportional to the square of the diameter of particles $[1,3]$.

Fig. 1 Dependence of the charge of coal particles of different sizes on their preheating. 1 to 5 - sizes of particles $0.5-0.25 ; 0.25-$ 0.175: $0.175-0.15 ; 0.15-0.1$ and $0.1-0.074 \mathrm{~mm}$

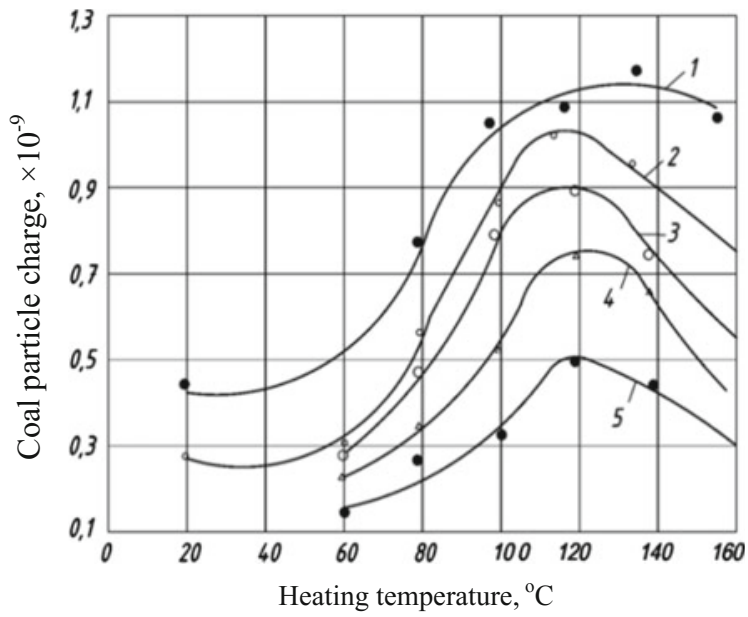


(a)

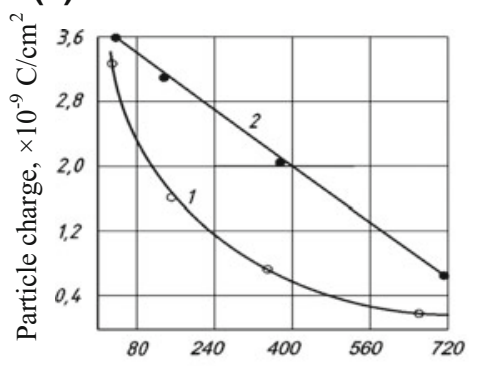

(b)

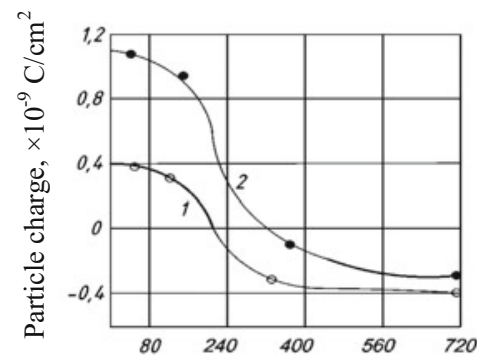

Fig. 2 Dependence of particle charge on particle sizes and the heating temperature. Particle size 1 particles have been air-dried; Particle size 2 particles have been heated up to $105{ }^{\circ} \mathrm{C}$

It is known that in case of dedusting and classification of bulk materials, including coal, the disadvantage of sieves with a mesh smaller than $0.15 \mathrm{~mm}$ is hole clogging, wearability, expensiveness and low efficiency of sifting, excluding their commercial application. Therefore, corona separators used for dedusting and sizing of different materials are of practical interest [7,8]. Working with the materials with fractions from 5 to $40 \%$, sized less than $0.07 \mathrm{~mm}$, it was found that extraction of this fraction on drum-type corona separators reaches up to $99 \%$. When the content of fine fractions increases the number of treatment operations increases as wellwhen their content is from 5 to $20 \%$ one or two operations are required, when it is $20 \%$ and higher-three operations $[7,8]$.

The surface electrical conductivity of minerals (especially that of dielectrics and semiconductors) depends on the amount of adsorbed moisture which dramatically increases electrical conductivity. Thus, when the ambient relative humidity is from 15 to $55 \%$ the charge value of particles remains almost the same, when the humidity changes by more than $55 \%$ the value of the coal particle charge will decrease dramatically. When developing design solutions for efficient separation it is necessary to take into account peculiarities of the particle charge in semiconductors and dielectrics. Mineral conductors are well separated from conductors and nonconductors. It is more difficult to separate semiconductors from nonconductors (mineral components of brown coal) which can complicate HTCBC upgrading and requires the intensified process of formation of electric charges with their increased values. It has been determined that charged particles are separated in the inhomogeneous electric field as a result of interaction of electric and mechanical forces which requires reasoning for selecting HTCBC separation techniques $[1,9]$. In the course of theoretical and laboratory researches of triboelectrostatic upgrading of brown coal the following disadvantages were identified:

- reduced efficiency as compared to the corona-electrostatic process of upgrading;

- in operation of the separator and free settling of clean coal the surface of plate electrodes must be free of dust which deteriorates separation;

- the concentrate contains increased mineral components and the emerging tailings-increased organic components. 


\section{Method}

Triboadhesive separators process conductive and nonconductive, organic, and nonorganic finely milled minerals and materials. Thus, if the air humidity is more than $70 \%$ adhesion of microscopic particles is increased by capillary forces [4]. The upper limit of the size of particles, which can retain on the surface, differs in different conditions and may exceed $100 \mu \mathrm{m}$. The diversity of factors affecting adhesive interaction of coal particles indicates the complexity of the process concerned and the possibility of its adaptive management [7]. The triboadhesive method is limited by high energy consumption and necessity to operate the equipment $U=20-60 \mathrm{kV}$. Therefore, this method of upgrading of heat-treated crushed brown coal will be unprofitable. When assessing the possibility of reliable separation of brown coal particles in terms of semiconductors from dielectrics when using a triboelectrostatic, triboadhesive and corona-electrostatic methods of HTCBC separation drum-type corona-electrostatic method was selected as the most productive one. The studies of corona-electrostatic separators (CES) with horizontal and vertical drums showed that under equal conditions separators with a vertical drum are 2-3 times more productive. Therefore, to upgrade HTCBC it is recommended to design a CES with a vertical drum-collecting electrode. When developing design solutions of a CES with a vertical drum it is necessary to take into account the following:

- The polarity of a corona-producing electrode affects the operation of separators with a corona discharge. The breakdown voltage is higher with a negative corona than that with a positive corona which is to be grounded.

- When the linear speed of a drum in constant electric field intensity is increased, the efficiency of a separator can be decreased.

- The corona discharge emerges only in the inhomogeneous electric field in a small area near a thin conductor. This discharge does not extend to the opposite electrode and can be regarded as a partial gas breakdown [7].

- One of the factors influencing the charge of HTCBC particles, sufficient for separation, is the corona discharge current. The corona current depends on the shape of a corona-producing electrode, voltage applied and structural features of the area of the separator corona discharge [10].

These dependences (Fig. 3a) for the corona current are applied to the simplest cases when the current is determined only by the voltage across the electrodes, their dimensions and ion mobility, thus, without taking into account such factors as temperature, pressure, humidity, gas velocity and type and the presence of suspended particles. Field intensity increases near a corona-producing electrode and remains almost the same in the rest interval between electrodes (Fig. 3b). To avoid sparking between cylindrical electrodes certain ratio between the wire radius $r$ and the cylinder radius $R$ must be ensured. For gas ionization without short circuit $R: r \geq 2.7$.

Based on the analysis of works of Russian and foreign scientists devoted to the charge and dynamics of separation of mineral particles in the course of 
(a)

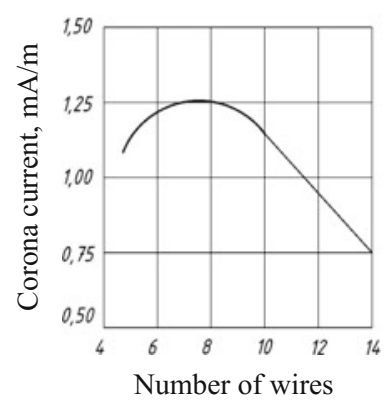

(b)

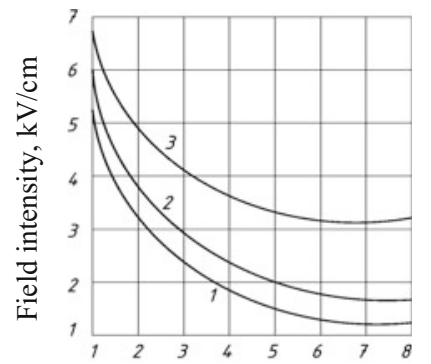

Distance from the corona electrode,

Fig. 3 a Dependence of the corona current on the number of corona wires [23]. b The curves of field intensity distribution at different current values with the corona discharge between cylinders (cylinder $\emptyset 148 \mathrm{~mm}$, wire $\emptyset 2 \mathrm{~mm}$ ) [7]. $1 U_{0}=26 \mathrm{kV}, I=0.67 \times 10^{-6} \mathrm{~A} / \mathrm{cm} ; 2 U_{0}=30 \mathrm{kV}$, $I=1.51 \times 10^{-6} \mathrm{~A} / \mathrm{cm} ; 3 U_{0}=38 \mathrm{kV}, I=10^{-6} \mathrm{~A} / \mathrm{cm}$

corona-electrostatic separation $[1-3,5,9,11-14]$, one can conclude that mineral particles in the electric field are separated by means of retaining of charged particles on the surface of the grounded rotating electrode (retaining mode) or as a result of their turning towards the electrode with the potential opposed to that of particles (extraction mode).

The above equations characterize the qualitative behavior on a CES horizontal collecting electrode of spherical particles and allow to assess the degree of influence of certain physical factors on the separation process [15-17].

Separation of HTCBC particles in a CES with a vertical drum is similar to that in a separator with a horizontal drum $[1,3,7,18-20]$. Unlike the diagram of forces in a separator with a horizontal drum, projection of the gravity force affecting the particle in a separator with a vertical drum, the axis of interaction of electrical pressing forces and a centrifugal separating force is equal to zero [18].

The effect of forces on particles retaining on the surface of a vertical collecting electrode

$$
m \frac{\mathrm{d} \bar{V}}{\mathrm{~d} t}=\sum \bar{F}=\bar{F}_{\mathrm{C}}+\bar{F}_{\text {mir. }}+\bar{F}_{\text {pond }}+\bar{F}_{\text {ad. }}+\bar{F}_{\text {c.f. }}
$$

where $\bar{F}_{\mathrm{k}}$ is the Coulomb force of the effect of the electric field on the charged particle

$$
F_{\mathrm{C}}=Q_{\mathrm{e}} E_{\mathrm{c}}
$$

where $Q_{\mathrm{e}}$ is the equilibrium particle charge in the operating area of a corona-producing electrode; $E_{\mathrm{c}}$ is zero tension of the corona discharge near a collecting electrode. 


$$
F_{\mathrm{C}}=4 \pi \varepsilon_{0} \cdot\left(1+2 \frac{\varepsilon_{1}-1}{\varepsilon_{1}+2}\right) \cdot r^{2} \cdot E^{2} \cdot f\left(R^{\prime}\right)
$$

$\bar{F}_{\text {c.f. }}$ is the centrifugal force affecting the particle retaining on the surface of a collecting electrode (drum) and is caused by rotation of the latter.

$$
F_{\text {c.f. }}=m \cdot v^{2} / R
$$

where $v$ is a linear speed of drum rotation,

$$
v=2 \pi \cdot R_{1} \cdot n / 60
$$

where $R_{1}$ is the radius of a collecting electrode; $n$ is the number of drum revolutions per minute

$R$ is the distance from the drum center to the particle gravity center,

$$
R=R_{1}+r_{1},
$$

where $r_{1}$ is the distance from the drum surface to the particle gravity center. Since $r_{1}$ is very little as compared to $R_{1}$, we can assume that $R_{1}=R$,

For spherical particles the weight is

$$
m=4 / 3 \pi \cdot r^{3} \gamma_{\mathrm{p}}
$$

Equation (12) with consideration of (5) and (6) is as follows

$$
F_{\text {c.f. }}=\frac{\pi^{3} \cdot r^{3} \cdot \gamma_{p} \cdot R_{1} \cdot n^{2}}{675}
$$

$\bar{F}_{\text {mir. }}$ is the mirroring force resulting from interaction of a particle resulting charge and induced to a collecting electrode of the electric charge equal in value to the resulting charge but opposed to it in its sign [10]. It is known that the particle discharge to the grounding surface of a drum is expressed in the equation [21]

$$
Q_{\mathrm{RES}}=Q_{\mathrm{e}} \cdot e^{-\frac{t}{R^{\prime} \cdot c}}
$$

where $Q_{\mathrm{RES}}$ is the residual charge after $t$ sec after a particle leaves the operating area of a corona-producing electrode; $Q_{\mathrm{e}}$ is the equilibrium particle charge which it receives in the corona field; $R^{\prime}$ is the contact resistance between a particle and a collecting electrode; $C$ is the space between a particle and an electrode.

$R$ and $C$ values determine the time constant of charging and discharging of a particle $\tau$ and depend only on the properties of a particle itself and do not depend on the external field intensity [22] 


$$
\tau=\varepsilon_{0} \cdot \frac{\varepsilon_{1} d_{\mathrm{a}}+\varepsilon_{2} \cdot\left(1-d_{\mathrm{a}}\right)}{\rho_{v_{1}} \cdot d_{\mathrm{a}}+\rho_{v_{2}} \cdot\left(1-d_{\mathrm{a}}\right)}
$$

for the air at $p v_{2}=0$ and $\varepsilon_{2}=1$ this formula changes to [22]

$$
\tau=\varepsilon_{0} \frac{\varepsilon_{1}+1-d_{\mathrm{a}}}{d_{\mathrm{a}} \cdot \rho_{v_{1}}}
$$

where $\varepsilon_{1}$ is dielectric conductivity of the particle material; $d_{\mathrm{a}}$ is the depolarization coefficient determined by the ratio of main ellipsoid axises $a, b, c$ (particle of a rotational ellipsoid shape); for a spherical particles $d_{\mathrm{a}}=0.5 ; p v_{1}$ is the specific bulk electrical conductivity of a particle which can be derived using the formula $p v_{1}=1 / p v$, where $p_{v}$ is the bulk resistance of the particle material.

Taking into account particle discharge in the BC area based on Eq. (9) the formula for the mirroring force when the particle leaves the ionization area is as follows:

$$
F_{\text {MIR. }}=\frac{\left(Q \mathrm{p} \cdot \mathrm{e}^{-\frac{t}{R^{\prime} \cdot c}}\right)^{2}}{16 \cdot \pi \cdot \varepsilon_{0} \cdot r^{2}}=\pi \cdot \varepsilon_{0} \cdot\left(1+2 \frac{\varepsilon_{1}-1}{\varepsilon_{1}+2}\right)^{2} \cdot r^{2} \cdot E_{\kappa}^{2} \cdot f^{2}(R) \cdot \mathrm{e}^{-\frac{2 t}{R^{\prime} \cdot c}}
$$

In practice, components $-\frac{2 t}{R c}$ of Eq. (20) are neglected since the value is close to 1 [14]. In separation of particles larger than $0.05 \mathrm{~mm}$ in air by the medium resistance force, adhesive force, Archimedes (buoyant) force, the ponderomotive force can be neglected, the equation of balance of forces for a separator with a vertical drum is as follows [21]:

$$
\sum \bar{F}=\bar{F}_{\mathrm{c}}+\bar{F}_{\text {mir. }}+\bar{F}_{\text {c.f. }}
$$

Equation for the resulting force [1, 9, 17, 23]

$$
\sum F_{\mathrm{res}}=\pi \varepsilon_{0} \cdot r^{2} \cdot E_{C}^{2}\left(1+2 \frac{\varepsilon_{1}-1}{\varepsilon_{1}+2}\right)\left(5+2 \frac{\varepsilon_{1}-1}{\varepsilon_{1}+2}\right)-\frac{\pi^{3} \cdot r^{3} \cdot \gamma_{p} \cdot R_{1} \cdot n^{2}}{675}
$$

With $\sum F_{\text {res }} \geq 0$ particles will be retained on a vertical collecting electrode, and with $\sum F_{\text {res }}<0$ they will be removed from the drum surface by the centrifugal force.

Therefore, the dependence of the size of nonconductor particles retained on a vertical collecting electrode in the area of the corona discharge:

$$
r=\frac{675 \varepsilon_{0} \cdot r^{2} \cdot E_{\mathrm{K}}^{2}\left(1+2 \frac{\varepsilon_{1}-1}{\varepsilon_{1}+2}\right)\left(5+2 \frac{\varepsilon_{1}-1}{\varepsilon_{1}+2}\right)}{\pi^{3} \cdot r^{3} \cdot \gamma_{4} \cdot R_{1} \cdot n^{2}},
$$

For example, for quartz particles with the density of $\gamma_{\mathrm{p}}=2.65 \times 10^{3} \mathrm{~kg} / \mathrm{m}^{3}$, dielectric conductivity $\varepsilon_{1}=4.5$, with field intensity of the corona discharge $E_{\mathrm{c}}=5 \times 10^{5} \mathrm{~V} / \mathrm{m}:$ 


$$
r=\frac{0.721}{R_{1} \cdot n^{2}}
$$

The content of the mineral component of HTCBC corresponds to the group of inertinite microcomponents-clay, sulfate sand, pyrite, and carbonates, i.e., analogue of quartz sand.

A number of HTCBC properties are similar to those of quartz sand: dielectric conductivity 4.5 , density of up to $1.500 \mathrm{~kg} / \mathrm{m}^{3}$, bulk density of up to $1.4 \mathrm{t} / \mathrm{m}^{3}$, surface area of up to $5 \mathrm{~m}^{2} / \mathrm{kg}$, and the diameter of a quartz sand particles is from $0.05 \mathrm{~mm}$ to $1 \mathrm{~mm}$ - with fraction particles "- $1 \mathrm{~mm}$ " of HTCBC with the highest content of non-combustible components. The purpose of this work is to study the principles of separation of non-combustible components of HTCBC, the results of investigation of the quartz sand model can be used for the analysis of the quality of upgrading of HTCBC on a drum-type CES.

In the mode of retaining nonconductor particles when a particle leaves the corona area, only the mirroring force $\left(\bar{F}_{\text {mir. }}\right)$ and the centrifugal force $\left(\bar{F}_{\text {c.f. }}\right)$ affect the latter [21]:

$$
\begin{gathered}
\sum \bar{F}_{\text {res }}=\bar{F}_{\text {mir. }}+\bar{F}_{\text {c.f. }} \\
\sum F_{\text {res }}=675 \cdot \varepsilon_{0} \cdot r^{2} \cdot E_{c}^{2}\left(1+2 \frac{\varepsilon_{1}-1}{\varepsilon_{1}+2}\right)^{2}=\pi^{3} \cdot r^{3} \cdot \gamma_{p} \cdot R_{1} \cdot n^{2},
\end{gathered}
$$

For quartz particles with the density of $2.65 \times 10^{3} \mathrm{~kg} / \mathrm{m}^{3}$ and dielectric conductivity $\varepsilon_{1}=4.5$, the field intensity of the corona discharge is $E_{\mathrm{c}}=5 \times 10^{3} \mathrm{~V} / \mathrm{m}$

$$
r=\frac{0.247}{R_{1} \cdot n^{2}}
$$

By comparison, the size of quartz particles outside the corona area in separators with a horizontal drum is as follows:

$$
r=\frac{0.247}{R_{1} \cdot n^{2}+895.44}
$$

\section{Results}

Calculation of the size of quartz sand particles retained on a vertical collecting electrode and on a horizontal electrode is shown in Figs. 4 and 5 for different values of the angular velocity of a collecting electrode and the linear speed of a drum [4]. Increase in size of nonconductor particles retained on a vertical drum, as compared to a horizontal drum at the same linear speed, is due to change of the centrifugal force affecting the particles on a vertical drum based on Eq. (12). 


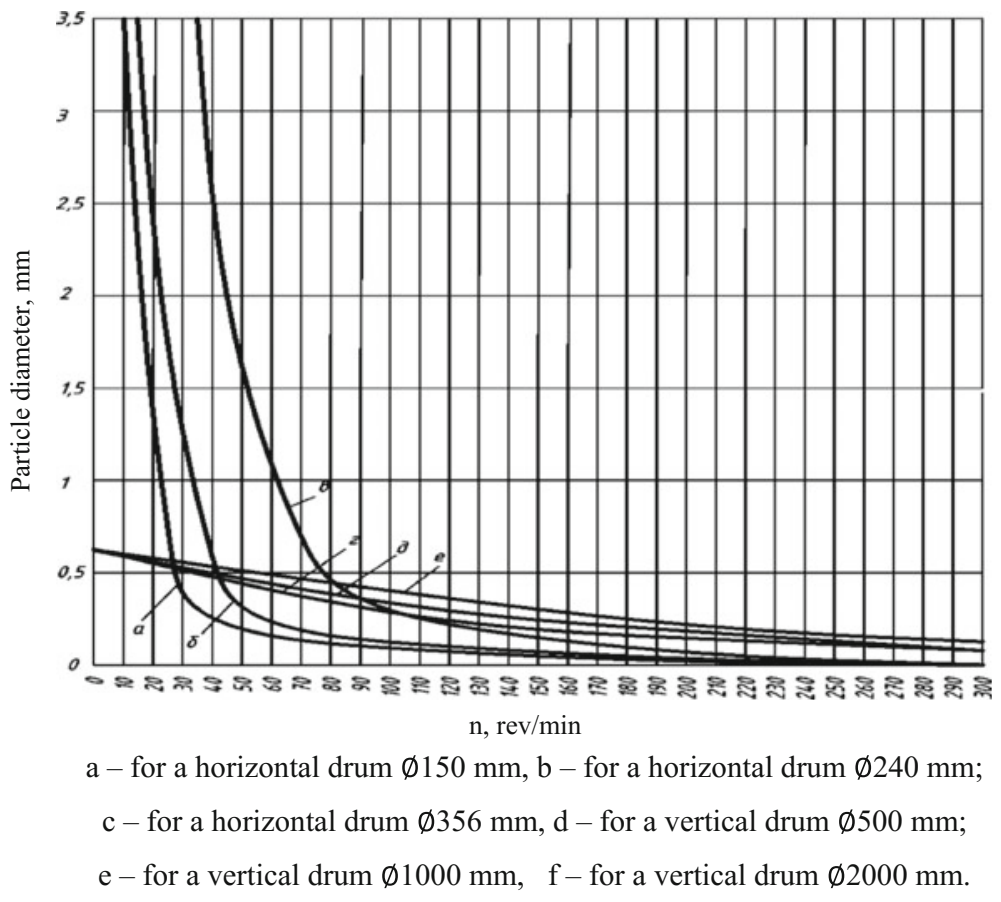

Fig. 4 Dependence of the diameter of retained particles outside the corona on the number of revolutions of a collecting electrode

The efficiency of a drum-type CES with a vertical collecting electrode can be determined based on the value of the efficiency of an electric separator with a horizontal electrode according to the formula [24]:

$$
Q_{\mathrm{V} . S .}=\sqrt{\frac{R_{V}}{R_{H}} \cdot \frac{L_{V}}{L_{H}}} \cdot N \cdot C_{\mathrm{v}} \cdot Q_{\mathrm{H} . S .},
$$

where $C_{\mathrm{v}}$ is the coefficient of occupation of the surface of a vertical collecting electrode which is determined by the ratio of the length of an outlet slot of the feeder ( $L$ o.f) to the length of the generator of a collecting electrode $(L \mathrm{v})$ in unit fractions $(C \mathrm{v}=L$ o.f./ $L \mathrm{v}) ; Q_{\mathrm{H} . \mathrm{S}}$ is the efficiency of an electric separator with a horizontal collecting electrode calculated by the formula $Q_{\mathrm{SEP}}=N \cdot L \cdot b \cdot q \cdot 3.6 \times 10^{3}$ $\mathrm{m} /$ hour) or experimentally, $\mathrm{t} / \mathrm{h} ; R_{\mathrm{V}}$ and $R_{\mathrm{H}}$ are the radiuses of a collecting electrode, vertical and horizontal, $\mathrm{m} ; L_{\mathrm{V}}$ and $L_{\mathrm{H}}$ are the lengths of the generator of a collecting electrode, vertical and horizontal, $\mathrm{m} ; N$ is the number of individual sections of an electric separator with a vertical collecting electrode (Fig. 6). 


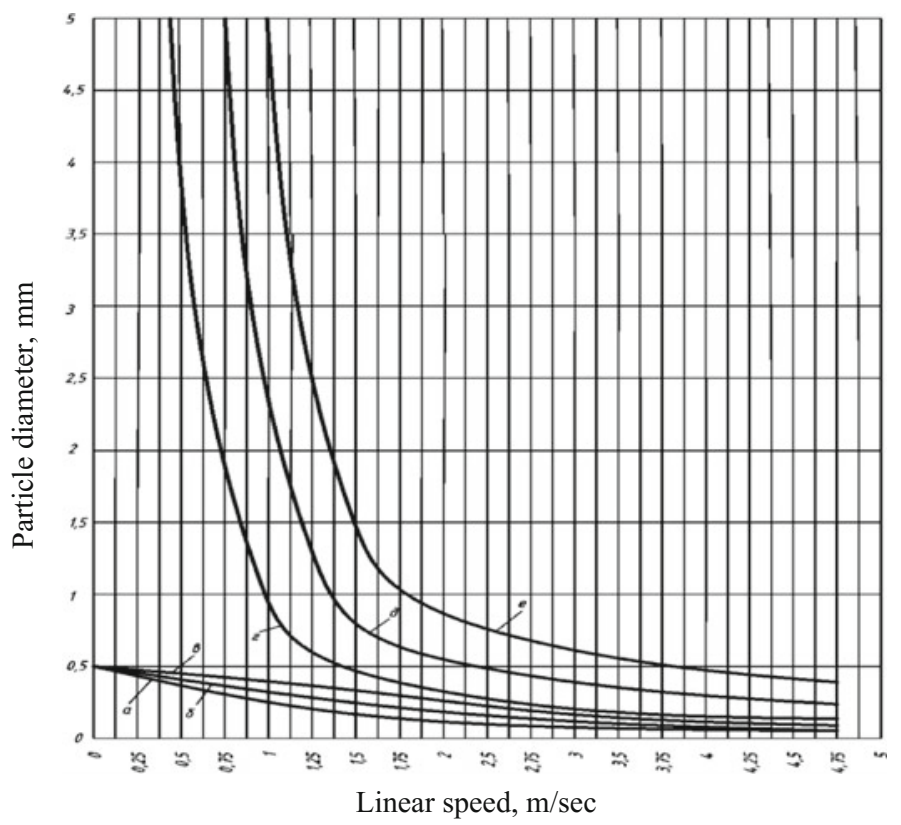

a - for a horizontal drum $\emptyset 150 \mathrm{~mm}, \mathrm{~b}$ - for a horizontal drum $\emptyset 240 \mathrm{~mm}$;

$\mathrm{c}$ - for a horizontal drum $\emptyset 356 \mathrm{~mm}, \mathrm{~d}$ - for a vertical drum $\emptyset 500 \mathrm{~mm}, \mathrm{e}$ - for a vertical drum $\emptyset 1000 \mathrm{~mm} ; \mathrm{f}$ - for a vertical drum $\emptyset 2000 \mathrm{~mm}$.

Fig. 5 Dependence of the diameter of particles retained outside the corona on the linear speed of a collecting electrode

When the linear speed of drums at constant intensity increases, the efficiency of the electric field of separators decreases. This is due to the increase in the centrifugal force - the size of retained particles is decreased, the size of the layer on the drum is decreased, separator efficiency is reduced.

Researches on classification and upgrading were performed using an experimental facility. To retain a collecting electrode of dielectric particles on the surface when the linear speed of a drum is increased the charging process for a material being separated must be [24]. To successfully separate mineral mixtures in a CES the particles must be charged and must contact the surface of a collecting electrode. The flow of ions generated in the "corona hood", affected by the electric field, moves to the collecting (grounded) electrode, where it charges the particles. Thus, the corona discharge current and its value are the factors affecting generation of charges on particles, which are sufficient for their separation. Separation of minerals in drum-type corona separators is determined by the discharge current intensity. However, the current levels of the corona discharge in different points of a collecting electrode differed and in the course of the experiment on a chamber corona separator the following trend was observed: the content of magnetite in the center of 
Linear speed of a horizontal drum, $\mathrm{m} / \mathrm{sec}$

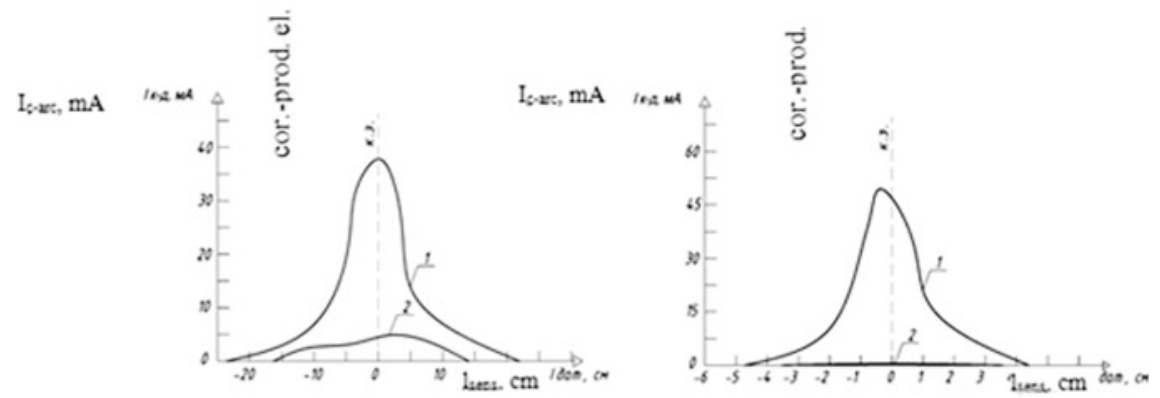

1 - voltage across a corona-producing electrode of $32 \mathrm{kV} ; 2$ - voltage across a coronaproducing electrode of $16 \mathrm{kV}$

Fig. 6 a Level and distribution of the current of the corona discharge on the surface of a vertical collecting electrode of $\emptyset 1 \mathrm{~m}$ for the "wire-drum" open system. b Level and distribution of the current of the corona discharge on the surface of a vertical collecting electrode of $\emptyset 1 \mathrm{~m}$ for the "wire-drum" open system bounded by glass-cloth plates in two sides

a section of the grounded electrode is usually minimal, it increases near the edges and then it decreases again [25]. Electrical resistivity of brown coal in its nature is ionic and is widely used depending on humidity. Heat-treated crushed brown coal has low electrical resistivity of $10^{-2}-10^{-4} \mathrm{Ohm} / \mathrm{m}$, similar to magnetite values, which allows to use the results of experiments for a comparative analysis (Fig. 7).

Fig. 7 Level and distribution of the current of the corona discharge on the surface of a vertical collecting electrode with the diameter of $1 \mathrm{~m}$ for the "wire-drum" system bounded by a glass-cloth plate in one side

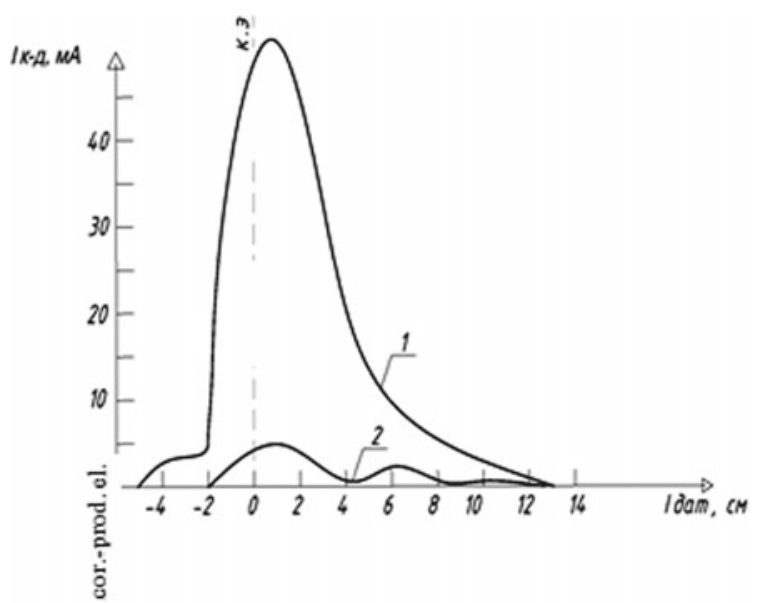

$\mathrm{I}_{\mathrm{c}-\mathrm{arc}}, \mathrm{mA}$

1 - voltage across a corona-producing electrode of $32 \mathrm{kV} ; 2$ - voltage across a corona -producing electrode of $16 \mathrm{kV}$ 


\section{Discussion}

Thus, the value and distribution of the current of the corona discharge on the surface of a collecting electrode affect the results of electric separation [8] (Fig. 7).

\section{Conclusion}

The upgrading of heat-treated brown coal will result in increased energy efficiency of enterprises of up to $5 \%$. The conducted researches proved that the effective techniques for separation of semiconductor (organic component) and dielectric materials (mineral component) for upgrading of heat-treated crushed brown coal are electric separation techniques including drum-type corona-electrostatic, plate-type electrostatic, drum-type triboelectrostatic and drum-type triboadhesive ones. The maximum level of coal particle charge was provided in a corona-electrostatic separator, a lower charge level was observed in a triboelectrostatic separator and the lowest ones were established in a plate-type electrostatic separator. Laboratory tests performed to establish the optimal size of HTCBC fed into separators and to select the most efficient upgrading technique showed the following:

- Techniques using a plate-type electrostatic separator and a triboadhesive separator work poorly for separation of heat-treated crushed brown coal.

- A drum-type CES is the most efficient one.

The basic efficiency constraint for drum-type corona-electrostatic separators is the diameter of a collecting electrode and one operating area. To select the directions for increasing the productivity and efficiency in separating heat-treated crushed brown coal in drum-type corona-electrostatic separators the results of studies of changes in force vectors affecting its charged particles, nature of their motion in the electric field resulting in separated products - organic and mineral components of brown coal, taking this into consideration the factors of the heating temperature, voltage across a corona-producing electrode (drum), diameter of a corona-producing electrode and its rotation speed, have been analyzed.

- Increase in the diameter of a corona-producing electrode results in reduction in the corona discharge current.

- Increase in the voltage across a corona-producing electrode of up to $32 \mathrm{kV}$ has the greatest influence on the yield of nonconductors.

- The rotation speed of a drum affects the quality of fractions separated, halving of the drum rotation speed (from 60 to $30 \mathrm{rev} / \mathrm{min}$ ) results in increase in the yield of a nonconductor fraction.

The increased efficiency of a drum-type CES is associated with removing a constraint from design parameters - the diameter of a collecting electrode (drum). It 
is realized through the change of orientation of a collecting electrode from horizontal to vertical.

Acknowledgments Researches are carried out with the financial support of the state represented by the Ministry of Education and Science of the Russian Federation. Agreement No. 14.579.21.0036, 05. Jun 14. Unique project Identifier: RFMEF157914X0036.

\section{References}

1. Olofinsky, N.F., Novikov, V.A.: Tribo-Adhesive Separation. Nauka, Moscow (1974)

2. Fraus, F. (1962). Electrostatic Separation of Granular Materials. U.S. Dept. of the Interior Bureau of Mines

3. Mesenyashin, A.I.: Electric Separation in Strong Fields. Nedra, Moscow (1978)

4. Simon, A.D.: Dust and Powder Adhesion, 2nd edn. Revised and enlarged. Khimiya, Moscow (1979)

5. Volkova, E.V., Zhus, G.V., Kuzmin, D.V.: Dielectric Separation of Various Polyconcentrates and Materials. Nedra, Moscow (1975)

6. Lower-James, E.: Fundamentals of electrical concentration of minerals. Mines Mag. 1(50) (1966)

7. Plaksin, I.N., Olofinsky, N.F.: Electric corona separation and its application for upgrading, classification and dedusting. In: Energy and Magnetic Separation Methods. Moscow (1965)

8. Kovalev, A.P.: Electrical Upgrading of Coal Dust. Works of the MPEI, 6th edn. (1950)

9. Vereshchagin, I.P., Levitov, G.Z.: Fundamentals of Electro-Gas Dynamics of Disperse Systems. Energia, Moscow (1974)

10. Olefinsky, N.F.: Electric separation of black sands of foundries. J. Mech. Eng. 12 (1947)

11. Egorov, V.L.: Magnetic, Electric and Special Methods of Upgrading of Ores. Nedra, Moscow (1977)

12. Karnaukhov, N.M.: Techniques for Upgrading of Bulk Concentrates using Electric Separation. Nedra, Moscow (1966)

13. Mamedov, A.I.: Physical Methods of Upgrading of Ore Stones. STI of NIITEKHIM, Moscow (1979)

14. Angelov, A.I., Ershov, V.S., Losaberidze, S.I., Pashni, M.M.: Movement of Charged Particles in the Electrostatic Field of a Drum-Type Corona-Electrostatic Separator. Electronic Materials Processing, 4 (1978)

15. Bachkovsky, M.V., Besov, B.V., Novikov, V.A., Olofinsky, N.F.: Electrical conductivity of materials and establishment of electrical resistivity. In: Theory and Practice of Techniques for Electric and Magnetic Separation of Minerals. Moscow (1968)

16. Urvantsev, A.I., Shikhov, N.V., Mushketov, A.A.: On the increase in Capacity of Drum-Type Corona-Discharge Electrostatic Separators. Upgrading of Ores. Special Issue for the XXI IMPC, pp. 62-65 (2000)

17. Cherchintsev, V.D.: Investigation of the triboelectric effect of minerals of dielectrics in their Corona separation. Works Magnitogorsk Inst. Min. 112, 119-122 (1975)

18. Levitov, V.I.: Alternating current Corona. Issues of the theory of research methods and theoretical characteristics. Synopsis of a thesis. MPEI, Moscow (1966)

19. Degtyarenko, A.V., Kashkarov, I.F.: Role and capabilities of electric separation in upgrading of complex titanic ores. In: Improvement of Electric Separation Processes and Concentration of Electric Separators. Interdepartmental Collection of Scientific Papers. Mechanobr (1987)

20. Khopunov, E.A., Revnivtsev, V.I.: Application of modern electrophysical methods in investigation of upgrading process. Works Uralmekhanobr Inst. 18, 54-62 (1972) 
21. Urvantsev, A.I., Shikhov, N.V., et al.: Development of a high-performance drum-type corona-electrostatic vertical separator. Tsvetnyie Metally 11, 71-74 (1995)

22. Kakovsky, I.A., Revnivtsev, V.I.: On the influence of the surface state in the process of electric separation of minerals with low electrical conductivity. In: 5th International Congress on Mineral Processing, Moscow (1962)

23. Komlev, A.M., Urvantsev, A.I., Shikhov, N.V., Zhuravsky, I.V.: Technological Tests of an Electric Separator SE-70/100: Report of the Uralmekhanobr Institute, p. 69. Sverdlovsk (1986)

24. Shikhov, N.V., Urvantsev, A.I. Electric separation as one of promising methods of complex processing of minerals. Abstracts of the Scientific and Technical Conference "Problems of Complex Processing of Titanomagnetite Ores of the South Urals", Magnitogorsk (2001, March 28-29)

25. Karnaukhov, N.M., Tarasova, T.B.: On increasing the efficiency of electric separators. Tsvetnyie Metally 4, 41-47 (1963)

26. Belov, P.D.: Electrostatic Fluidization Separation. Nedra, Moscow (1977)

27. Komlev, A.M., Urvantsev, A.I., Druzhinin, V.M.: Investigation of Physical Models of Separation in Electric Separators: Report of the Uralmekhanobr Institute, p. 66. Sverdlovsk (1984)

28. Urvantsev, A.I., Shikhov, N.V., Zaitsev, G.V.: Results of researches and practice of upgrading of minerals by means of electric separation. News Higher Educ. Inst. Min. J. 5, 37-51 (2005)

29. Revnivtsev, V.I., Olofinsky, N.F.: Current state and development trends of electrical separation of minerals. In: Works of the World Electrotechnical Congress. VELK Organizing Committee, Moscow (1977)

30. Meseniashin, A.I.: On the effect of electric forces on particles at the electrode. Electron. Mater. Process. 5, 65-69 (1982)

31. Plaksin, I.N., Olofinsky, N.F.: On application of corona dedusters and classifiers for upgrading of fine sizes of minerals. In: Application of the Electric Field Forces in Industry and Agriculture, pp. 239-250. Moscow (1964)

Open Access This chapter is licensed under the terms of the Creative Commons Attribution 4.0 International License (http://creativecommons.org/licenses/by/4.0/), which permits use, sharing, adaptation, distribution and reproduction in any medium or format, as long as you give appropriate credit to the original author(s) and the source, provide a link to the Creative Commons license and indicate if changes were made.

The images or other third party material in this chapter are included in the chapter's Creative Commons license, unless indicated otherwise in a credit line to the material. If material is not included in the chapter's Creative Commons license and your intended use is not permitted by statutory regulation or exceeds the permitted use, you will need to obtain permission directly from the copyright holder.

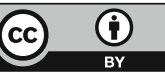

